\title{
The industrial robot is in conjunction with homework and system integration
}

\author{
Zhi Wei Tang \\ Xiamen nanyang vocational College , Fujian 361000 , China \\ 1063796772@qq.com
}

Keywords: Industrial Robot, Cooperation, Modbus-TCP, System Integration, Intelligent Factory .

\begin{abstract}
Along with German industry 4.0 and China makes 2025 wait planned implementation, Internet + , the thing unites a net, intelligence factory, the rise of the unmanned factory, being in conjunction with of various industrial robotses homework is foundation.Previously enter to make realm, intelligence's making system and gentle manufacturing system is the developments of the modern industry inevitable trend.Introduce currently in the intelligence manufacture industry in the middle of the system in common use industrial robot, the intelligence transports a car, stereoscopic warehouse, the sense of vision spreads feeling to identify to wait sub- system.These sub- systems pass Siemens PLC MODBUS-TCP agreement, mutual conjunction together, the many sets industrial robot carries on mutual correspondence to attain many work prefaces to be in conjunction with a homework, and carried on design analysis to position and sport status model of industrial robot, and of each mold piece system integration of carry out process.Lays the foundation for constucting intelligence factory and unmanned factory.
\end{abstract}

\section{Introduction}

The intelligence makes a technique is make a technique and automate technique, the system academicses, such as engineering and artificial intelligence...etc. to permeate each other, mutual intertexture but a comprehensive technique of formation.It concretely expresses for:the intelligence design, the intelligence process, the robot operate, the intelligence control, the intelligence craft program, the intelligence adjust degree and management, intelligence to assemble, intelligence diagraph and diagnosis etc..The intelligence manufacturing is new development and importance that makes gentle automation of system and integration automation to constitute part, therefore the intelligence manufacturing will integrate toward the intelligence in the future of direction development.In common use function mold piece(sub- system) is as follows:

\section{Industrial robot}

Switzerland ABB limited company industrial robot IRB 2400:It is the biggest and load and put for 7 kgs, being iner common use than to weld, take.The robot constitutes to ${ }^{\circ}$ from two parts:Controller:and Manipulator.Integrated correspondence function.Is Japanese Anne the Chuan limited company MH12:Carry to weigh a $12 \mathrm{kgs}$, the arm exhibition $1440 \mathrm{mms}$, the accuracy 0.08 mms is iner common use than to assemble, assign, the equipments look after, material porterage, packing, blunt press etc., integrated to correspond by letter function.

\section{The sense of vision spreads feeling to identify}

The sense of vision identifies an ambulation type the Jian pack dish system for cent is gather a picture to obtain, the picture identify, picture fixed position, robot code Duo, dismantle a Duo and machine number to control to shuttle to run about and place of honor calculator management for the open type of the integral whole gentle automation. The system sense of vision identifies part to differently place noodles and place a direction to carry out sense of vision to identify a fixed position to the different product of the static state( the product of random emergence is transported to appointed website position) or the dynamic state( the product of random emergence the ambulation don't stop over in transporting a chain) and the same product of the static state or dynamic state.The product had already been identified and positioned is grabbed at according to the product type, position information choice tongs having been already acquired by the robot, again according to 
product the website position that need to be placed run about into the website, complete to the dismantling of product tasks such as Duo, porterage or code Duo etc. and finally carry out the automatic cent Jian of product to pack dish and go together with to send.

\section{China lately loosens an intelligence AGV}

Simple and transported AGV is the AGV system being simple to turn the series that the lately loose limited company provides according to different customer's need, satisfy car the intelligence logistics transport.Mainly be applicable to a car zero parts profession, electronics product and light industry zero transport.

\section{MODBUS-TCP corresponds by letter}

MODBUS-TCP make MODBUS_RTU negotiate Be circulated to an ether net, MODBUS TCP use TCP-IP and ether net at stand point transmission MODBUS report text, MODBUS TCP combined physical network of ether net and network standard TCP|IP and with MODBUS is the data that applies agreement standard to mean method.MODBUS TCP correspondence's reporting a text be sealed Be packed to an ether net TCP|the IP data wrap in.And traditional string of method, MODBUS TCP inserts MODBUS of a standard report text arrive TCP report in the text and no longer take data schools to check with address.

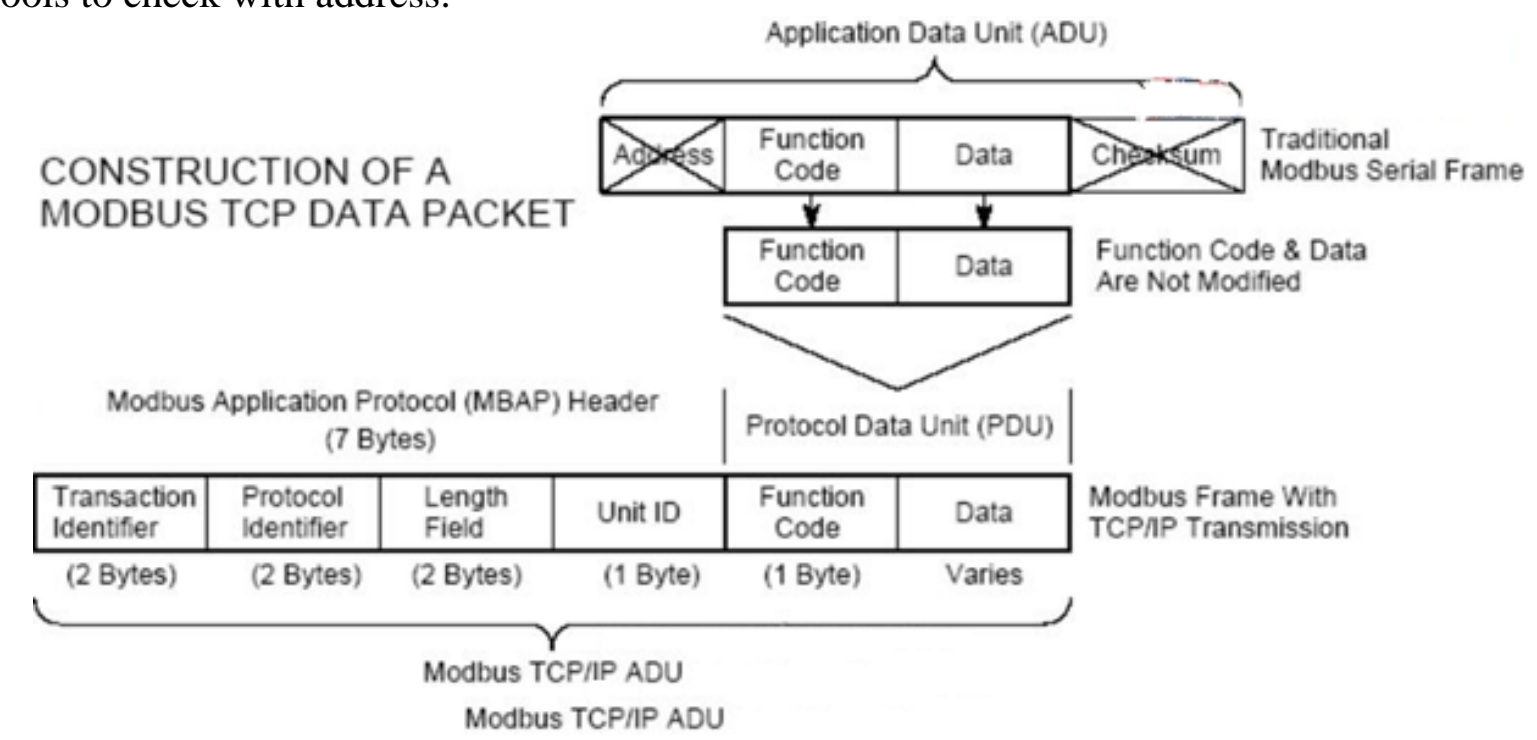

\section{Siemens PLC1200 correspondence function explains}

Siemens the S7-1200 is well contented with medium small scaled system need of automation.profinet of Siemens S7-1200 series integration connects to used for the communication that weaves distance, HMI communication and PLC.In addition it still negotiates to support through a liberal ether net with the communication of third square equipments.Should connect to take a RJ45 that has an auto to cross net line(auto-cross-over) function conjunction machine, provide 10|100 Mbits|s data baud rate, support Siemens S7-1200 cpus at most can add three communication molds piece.The string line communication that order for ordering of RS485 and RS232 communication mold piece provides a conjunction. To the set of the communication the Tai and plait distance adopted to expand instruction or database function and uss to drive agreement and Modbus RTU lord station and negotiate from the station, all include step the 7 Basic engineering set is at Siemens Tai system in.Negotiate:TCP-IP native, ISO-on-TCP and S7 communicationses.

\section{The many sets industrial robot is in conjunction with homework design analysis}

Many robot systems are in the function, structure and reply complicated, don't know, the dangerous environment and task etc. obviously surpass single industrial robot, but at the same time because of it complexity, dynamic state, change sex and Ou match the sexual increment also brings the design and the analysis some problems, therefore many robots of mutual the coordination harmoniously makes to seem to be to pass importance.Aim at a difference to reach a being in conjunction with of many robot systemses technical research problem.Build up a main robot 
position's fastenning for the sitting of original point mark, such as figure 1.Should sit mark with lord robot current position for ordered at first, at present direction is $\mathrm{x}$ stalk square to.The $\mathrm{t}$ in diagram is one time, the member robot just fastens the position in the xOy by mark BE.(x,y)Along with lord sport of the robot, its direction and positions all occurrence variety, arrive $\mathrm{t} 2$ times, sit mark to fasten renewal for the $\mathrm{xO}^{\prime} \mathrm{y}$.At renew of sit mark to fasten in, the position renewal of member's robot BE.(x', $\left.y^{\prime}\right)(\mathrm{T} 2-\mathrm{t} 1$ ) for by mark fasten to renew time to tread long, this period of time, inside the partition, lord the robot and member's robot all use xOy to sit mark to fasten.

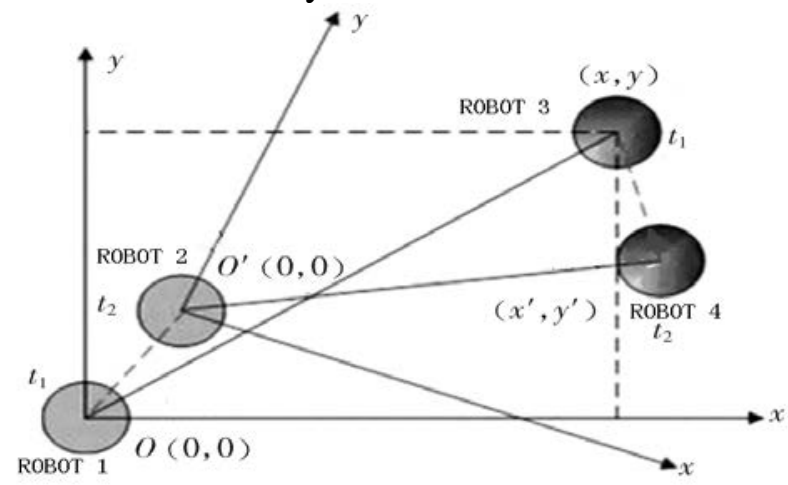

(Figure 1 sit mark conversion model)

$$
f\left(\boldsymbol{X}_{k}, \boldsymbol{u}_{k}\right)=\left[\begin{array}{c}
\boldsymbol{X}_{k+1}=f\left(\boldsymbol{X}_{k}, \boldsymbol{u}_{k}\right)+\boldsymbol{\omega}_{k}, \\
x_{k}\left(\sin \left(\theta_{k}+\Delta \theta_{k}\right)-\sin \theta_{k}\right) \\
y_{k}-\frac{\Delta D_{k}}{\Delta \theta_{k}}\left(\cos \left(\theta_{k}+\Delta \theta_{k}\right)-\cos \theta_{k}\right) \\
\theta_{k}+\Delta \theta_{k} \\
\Delta \theta_{k} \mid>0 .
\end{array}\right]
$$

(Formula 1 robot exercises status model)

Each robot professional enters process, such as figure 1 medium of $t 2$ - inside the partition, t's a time's each robot passes to blend a mileage to account and top instrument data, carry on an oneself fixed position.Predominant the robot carry on an examination to member's robot and pass to be like towards shooting to be like a mark settling, from the member the robot at shoot to be like the head of becoming and compute its projection in level direction and reckon a target position and shoot to be like a main light stalk thus of upward clip Cape $\eta$ at the square of level.According to double eyes sense of vision model, the calculability is main the distance of of the robot and member's robot.According to the $\eta$ 's Cape, can obtain from the laser scanner collect of the data be prognosticated the distance of target and predominance robot.The laser scanner passes to compute a member robot up two cylinder distances placing are bad, deduce the robot direction of a member.Pass to associately filter a model, blend each spread a feeling machine of prognosticate a data, estimate a member robot status information. The industrial robot exercises the status model as formula 1.

\section{MODBUS-TCP corresponds by letter the system integration of agreement}

system integrations including equipments system integration and application system is integrated:Equipments system integration, can also be called hardware system integration, point with build and organize the information-based management support platform in the organization for purpose.Application System Integration, take the height of system as customer's need to provide applied system mode, and carries out the concrete technique solution and operation project of the system's mode, fored a customer to provide an overall system solution.

The system integration designs diagram 


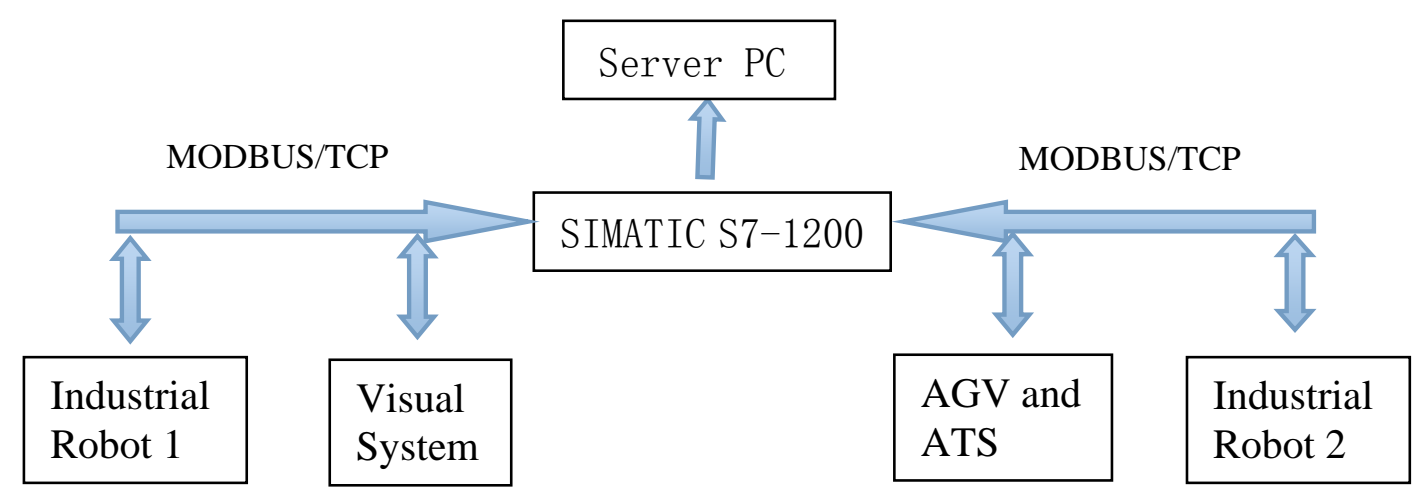

\section{MODBUS-TCP the correspondence negotiate the system integration carry out process}

Weave the distance software Step7 Basic V10.5.The Modbus database software options wraps.The S7-1200 makes a Modbus lord station, the S7-200 makes Modbus from stand.The S7-1200 is as follows the allocation step of Modbus lord station:Need to choose "Create new project" options first, then input Modbus_Master in"Project name:";At"Path:"The saving path that modifies item is "C:I";Click "Create", established the new item of a document Modbus so.Click "Project View" that the gate sees diagram left bottom Cape to cut over item to see bottom in the diagram, after opening, in "Devices" under the label, click "Add new device", at flick ofopen the input device in the menu"PLC_1" combine in the equipments list to choose a CPU type.PLC hardware set Tai:After inserting CPU, click the empty slot of CPU the left side, on the right side of find out in"Catalog"'Communication" under of RS485 mold piece, double click this, in"Port Configuration" can to the carrying on of port parameter allocation, the wave leads especially for:9600;Proved prescription type in the school:Have no;The data is:8;Stop:1;The hardware flows a control:Have no; Wait for time:1 ms establishes parameter.Write to send out procedure in PLC.See diagram in the item management the bottom double click "Device"- 》 "Program block"- 》 "Add new block" win election to choose "Organizations Block(OB)" at the window way flicking open, then in the window way of its the right side the choice"Startup", clean the options of "Symbolic Access Only" and hang up.Click "OK" future reunion to open this, then be starting to use MB_COMM_LOAD in OB piece.Edit and translate item:Can download procedure to PLC after editing and translating and having no mistake in, equally right shot PLC_1 item, at flick ofopen the menu choice"Download to Device".The S7-200 is Modbus from the allocation step of station as follows:"V4.0 steps the 7 MicroWIN SP6" diagram mark that clicks table's top, after opening and write Modbus from station procedure.

\section{Summary}

Passing the MODBUS-TCP correspondence agreement can carry out industrial robot of many sets to be in conjunction with homework and AGV to transport small car, sense of vision to identify system, stereoscopic warehouse and PLC work to control the machine each mold of etc. piece to carry on an information system integration and raise an industry production efficiency thus.

\section{References}

[1]RKurazume and SHirose.Study on cooperative positioning system [C].Proc. IEEE Int.Con.fRobot.Automat.,Leuven,Belgium,May,1998.

[2] ROUMELIOTIS S I, BEKEY G A. Distributed multiro-bot localization [ J ] . IEEE Transaction On Robotics And Automation,2002,18(5):781 - 795.

[3] M.A.René Maresch. Factories of the future: Mobile manipulators for aerospace production. 2013-04-29[2013-09-20]. 
[4] GanYaHui, Many mechanical arm coordinated control study were reviewed. Control and decision, 2013, 28 (3) : 321-333.

[5] Xu-dong Hu; Based on the network of heterogeneous industrial robot integration technology research [D]. Zhe jiang university; In 2004.

[6] Choi W, Pantofaru C, Silvio S. A general framework for tracking multiple people from a moving camera. IEEE Transactions on Pattern Analysis and Machine Intelligence,2013, 35(7): 1577- 1591.

[7]Lv Hong-Bo, Song Yi-Xu, Jia Pei-Fa. Incorporation of prior knowledge in adaptive learning for modeling the robotic pro-file grinding. Robot, 2011, 33(6): 641- 648. 